\title{
G-DEEC: GATEWAY BASED MULTI-HOP DISTRIBUTED ENERGY EFFICIENT CLUSTERING PROTOCOL FOR WIRELESS SENSOR NETWORKS
}

\author{
Sheenam \\ M. Tech Research Scholar \\ Department of Electronics and Communication Engineering, Punjabi University, Patiala, \\ India
}

\begin{abstract}
Wireless sensor network is composed of hundreds and thousands of small wireless sensor nodes which collect information by sensing the physical environment. The sensed data is processed and communicated to other sensor nodes and finally to Base Station. So energy efficient routing to final destination called base station is ongoing current requirement in wireless sensor networks. Here in this research paper we propose a multi-hop DEEC routing scheme i.e. G-DEEC for heterogeneous networks where we deploy rechargeable intermediate nodes called gateways in-between cluster head and base station for minimizing energy consumption by sensor nodes in each processing round thereby increasing the network lifetime and stability of wireless sensor networks unlike DEEC.
\end{abstract}

\section{KEYWORDS}

Wireless Sensor Networks, Heterogeneous Protocol, Gateway, DEEC, Energy Consumption.

\section{INTRODUCTION}

Wireless sensor networks can be viewed as a move leading in direction of wireless technology from wired one. These are network of tiny and cheap application-centric battery powered electronic devices called sensor nodes with on board sensing, processing and communicating capabilities connected to other nodes via Radio, infrared and optical media. The main function of these nodes is to sense the environment for temperature, humidity, pressure, intensity, pollutant levels, chemical concentrations etc. according to the application they are being installed for and report this gathered information after processing to Base Station. So routing of information from sensor nodes to Base Station with lesser consumption of battery is one of the current concerns in this field. Processing of data in network is cheaper than data transmission. In convention routing approaches like DT (Direct Transmission), sensor nodes directly send data to base station thereby nodes away would require more energy for routing information [1]. In MTE (Minimum Transmission Energy), sensor nodes near to base station die faster as they are more prone to a part communication. So there comes the concept of hierarchical routing using clustering where one sensor node having higher energy is considered as Cluster Head $(\mathrm{CH})$. This type of

DOI: $10.5121 /$ ijci.2015.4502 
communication is called single hop (i.e. transfer of information from sensor nodes to $\mathrm{CH}$ and then to base station) routing.

Many single hop routing protocols for homogeneous environment where each sensor nodes is equipped with same initial energy are LEACH (Low Energy Efficient Clustering Hierarchical LEACH), PEGASIS (Power-Efficient Gathering in Sensor Information Systems), TEEN (Threshold Sensitive Energy Efficient Network), HEED (Hybrid Energy-Efficient Distributed clustering) etc. And the single hop routing in heterogeneous environment where nodes are having different initial energy is done by SEP (Stable election protocol) and DEEC (Distributed Energy Efficient Clustering) etc. Many hierarchal routing schemes for saving the transmission distance have already been proposed for wireless sensor networks but still Cluster Head still suffers long distance transmissions. So here we propose a multi-hop routing protocol using intermediate rechargeable transmission devices known as gateway nodes between cluster head and base station as a defense mechanism for energy constraint battery powered sensor nodes prolonging the processing time of sensor nodes and also reducing the average transmission distance between sensor nodes and Base station.

The paper is organized as follows: Section 2 gives brief layout of related protocols in previous years; Section 3 provides detailed theory about proposed technique and model proposed for sensor network area. Section 4 includes the simulation results of network parameters; section 5 includes the conclusions and future scope of research.

\section{RELATED WORK}

Many different routing protocols have been already proposed for energy efficient routing for increasing the lifetime and stability of networks. Hierarchical routing includes clustering with $\mathrm{CH}$ selection on basis of initial energy. LEACH was the first routing protocol to introduce single-hop routing for reducing communication overhead by introducing the concept of clustering in homogeneous network field and selecting higher energy node as cluster head. There is randomized rotation of $\mathrm{CH}$ positions so as not to drain out battery of single sensor node. Simulation results showed reduction in energy consumption as compared to non clustered conventional Direct Communication protocol. Clustering concept was applied to all homogeneous protocols like LEACH [2], PEGASIS [3] and HEED [4]. CHs so formed in clustering perform fusion of aggregated data and pass this data to BASE station. All these homogeneous schemes perform badly in heterogeneous environment. The lower energy nodes die more frequently over higher energy nodes as there is no criterion for discriminating the nodes on energy basis. So here comes SEP [5] where the author considered two level heterogeneity and CH selected on basis of initial energy in each node. Nodes with more initial energy have chance to become $\mathrm{CH}$ than other nodes. Further advancement in criterion of $\mathrm{CH}$ selection is made in DEEC [6] where CHs are selected on basis of ratio of residual energy and average energy of each node. Nodes with higher residual energy are probable to be $\mathrm{CH}$ than other nodes. Advancements in DEEC was done in DDEEC [7] where advance nodes will have same probability of becoming $\mathrm{CH}$ as normal nodes when they are as same in energy to lower ones unlike DEEC where advance nodes continue to become $\mathrm{CH}$ even after energy depletion. Further E-DEEC (Enhanced DEEC) [8] proposed three level heterogeneity for DEEC algorithm where third kind of nodes called super nodes are been taken in network field. Simulation results showed E-DEEC performed better than SEP in terms of network lifetime. More over EE-TDLC [9] was proposed where two tier $\mathrm{CH}$ 
selections are proposed. At first tier data is transmitted from sensor nodes to primary $\mathrm{CH}$ selected on basis of residual energy then in second tier data is transmitted from primary $\mathrm{CH}$ to secondary $\mathrm{CH}$ selected from primary $\mathrm{CHs}$ then to base station or directly to base station depending to whichever is nearest. G-SEP [10] is advancement over SEP where CH is selected not only on initial energy basis as in SEP but with residual energy. SEARCH [11] is also a kind of advancement over DEEC which achieves decrease in time of transmission by frequent updating of $\mathrm{G}$ value i.e. available number of nodes for becoming $\mathrm{CH}$. The unfavorable situation exists in LEACH, SEP and DEEC where G value reduced to zero which is not updated until next round of transmission. So SEACH achieves better results in comparison to LEACH, SEP and DEEC. Further EEHC (Energy Efficient Heterogeneous Clustered Scheme) [12] for Wireless Sensor Networks, where the effect of heterogeneity of nodes in terms of their energy in WSNs that are hierarchically clustered has been studied. An energy efficient heterogeneous clustered system for WSNs based on weighted election probabilities of each node to become a cluster head according to their residual energy in each node is proposed. Simulation results showed the proposed heterogeneous clustering approach is more effective in prolonging the network lifetime compared with LEACH. In [13] author proposed TDEEC (Threshold Distributed Energy Efficient Clustering) protocol by following thoughts of DEEC and modifying the threshold value based on which the node decide to become $\mathrm{CH}$ or not as shown in equation below

$$
\mathrm{T}(\mathrm{n})=\left\{\begin{array}{cc}
\frac{\mathrm{p}_{\mathrm{i}}}{1-\mathrm{p}_{\mathrm{i}}\left[\mathrm{r} \times \bmod \left(\frac{1}{\mathrm{p}_{\mathrm{i}}}\right)\right]} * \frac{\text { residual energy of a node } * \mathrm{k}_{\mathrm{opt}}}{\text { average energy of network }}, \quad \text { if } \mathrm{n} \in \mathrm{G} \\
0 & \text { otherwise }
\end{array}\right\}
$$

In [14] authors' proposed heterogeneous SEP protocol (H-SEP) for increasing lifetime of heterogeneous wireless sensor networks. They discussed two types of sensor nodes i.e. normal and advanced. H-SEP increases the network lifetime and it is energy efficient than SEP. More packets are sent to the base station. In this 2-level heterogeneity is introduced in terms of the node energy. A significant improvement was observed in the lifetime because the number of rounds is more with 2-level H-SEP in comparison with SEP protocol. In [15] authors proposed Balanced and Centralized Distributed Energy Efficient Clustering (BCDEEC) for heterogeneous wireless sensor a network which is an extension of DEEC where each advanced node select itself to be gateway. BS is located at center of network field. Probability $\mathrm{P}_{\mathrm{g}}$ is such chosen that expected number of gateways is $\mathrm{K}_{\mathrm{g}}$ and probability of $\mathrm{Nm}$ advanced nodes becoming gateways is $P_{g}=\frac{K_{g}}{N m}$. Simulation results showed improvement in stability period compared to DEEC and SEP.

In [16] authors proposed performance evaluation of cluster-based routing protocols used in heterogeneous wireless sensor networks, authors analyzes the performance of Distributed energy efficient clustering (DEEC) protocol in terms of energy consumption, network lifetime and energy balancing. A new clustering protocol called Clustering Technique for Routing in wireless sensor networks (CTRWSN) has been proposed for prolonging the network lifetime. Simulation results shows that the lifetime of the proposed routing protocol is $40 \%$ longer than DEEC and showed that energy is well balanced as compared to DEEC. 
In [17] authors proposed Extended Stable Election Protocol which considers three levels of heterogeneity in terms of nodes initial energy termed as normal, intermediate and advance nodes in wireless sensor networks. In SEP-E assume a case where ' $b$ ' intermediate nodes equipped with ' $\mu$ ' times and ' $m$ ' advance nodes equipped with ' $\alpha$ ' times more energy than normal nodes respectively and modified their epoch (no of time a node to be $\mathrm{CH}$ in a round) for intermediate and advance nodes by modifying their election probabilities to be cluster head, to balance energy consumption of sensor nodes. Simulation settings and results showed that Enhanced SEP performs well than LEACH. It shows 1.94 times and 2.09 times improvement in terms of FDN and throughput.

In [18] authors introduced gateway based homogeneous energy-efficient routing protocol (MGEAR) for Wireless Sensor Networks (WSNs). The sensor nodes are divided into four logical regions on the basis of their location in the sensing field and Base Station (BS) is installed out of the sensing field and a gateway node at the centre of the sensing area. If the distance of a sensor node from BS or gateway is less than predefined distance threshold, the node uses direct communication and the rest of nodes are divided into two equal regions whose distance is beyond the threshold distance. Cluster heads (CHs) are selected in each region which is independent of the other region. Results showed that proposed M-GEAR outperforms LEACH in terms of network life time by $40 \%$ and also in terms of throughput by a factor of 5 .

In [19] authors proposed an improved algorithm called DEEC with Isolated Nodes (DEEC-WIN) where the mechanism of nodes joining a cluster is improved. Some nodes nearer to base station communicate directly without being part of any cluster. Simulation results showed DEEC-WIN consumes less energy in comparison to LEACH and DEEC which helps to extend the network lifetime.

In [20] authors proposed an Away Cluster Head Scheme (ACH) for energy efficient clustering protocols which make clusters even in number of nodes in each cluster so energy dissipation in each round is comparable and cluster head will be considered as normal node if the distance from nearest $\mathrm{CH}$ is less than $12 \mathrm{~m}$. Simulation results showed DEEC-ACH performs well than DEEC as the adjacent and very close nodes are made CHs in DEEC.

In [21] authors proposed Cluster Head Re-election Protocol (CRP) for heterogeneous wireless sensor networks where re-election of cluster heads is done from the elected ones having maximum residual energy which is taken as final cluster head. Simulation results shows $46 \%$ decrease in average residual energy as compared to LEACH and SEP.

In [22] authors proposed the new clustering algorithm based on multitier network topology to prolong the lifetime of wireless sensor network called Multitier Algorithm Protocol (MAP) where nodes of same energy transmit data through two tier CHs located at tier 1 around BS and tier 2 around tier 1 before they reach the Base station located at centre of network. CHs are selected based on fuzzy logic. Simulation results showed there are still 95 alive nodes up to 8000 iteration where SEP and DEEC with zero alive node up to 5000 iteration, thus prolonging the lifetime of network.

In [23] authors proposed a modified energy efficient protocol named as EM-SEP. In the research, author modified $\mathrm{CH}$ selection criteria by balancing energy consumption to increase lifetime of the 
International Journal on Cybernetics \& Informatics (IJCI) Vol. 4, No. 5, October 2015

network and also to increase stability period of WSN. Even authors worked on the concept if there is more than one $\mathrm{SN}$ available to become $\mathrm{CH}$ then it would pick the node having highest energy. Simulation results showed that EM- SEP performs 5\% in terms of stability period and 5\% in lifetime of network than SEP.

\section{NETWORK MODEL OF PROPOSED PROTOCOL (G-DEEC)}

In this research, we consider a $100 \mathrm{~m} * 100 \mathrm{~m}$ network field with $\mathrm{n}$ sensors deployed randomly in sensor field. Some assumptions for the proposed model shown in figure 1 are as:

- BS $(50,125)$ is deployed away from network field. Sensor nodes and base station are stationary after deployment.

- BS is aware about its position in network field and about location of sensor nodes with the help of GPS (Global positioning system).

- 9 gateway nodes are deployed at pre-planned distance in network field.

- Gateway nodes deployed systematically are stationary.

- We use heterogeneous nodes with different initial energy values.

- Sensor nodes deployed randomly over the sensor field.

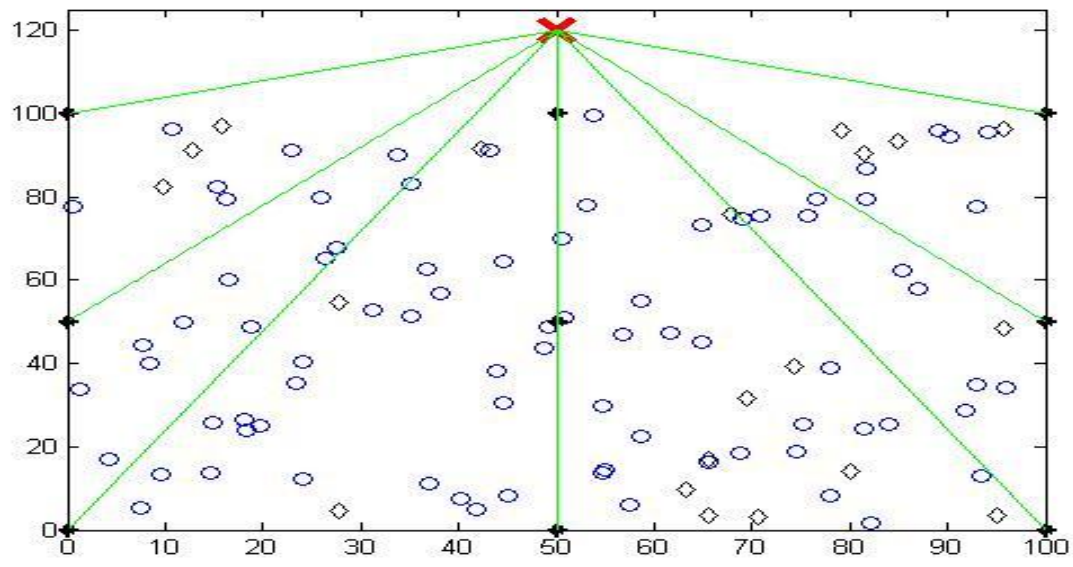

Figure1. Network model of G-DEEC $(100 \mathrm{~m} * 100 \mathrm{~m}$ field $)$

- Normal nodes

$\diamond \quad$ Advanced nodes

$\times \quad$ Base Station

* Gateway nodes

Here we used first order radio energy dissipation model proposed as in LEACH [2] shown in figure 2 where the transmitter dissipates energy to make the electronic circuit and power amplifier work. According to the propagation model the energy consumed in transmitting L-bit message with achievable value of signal to noise ratio over distance $d$ between transmitter and receiver is given as 


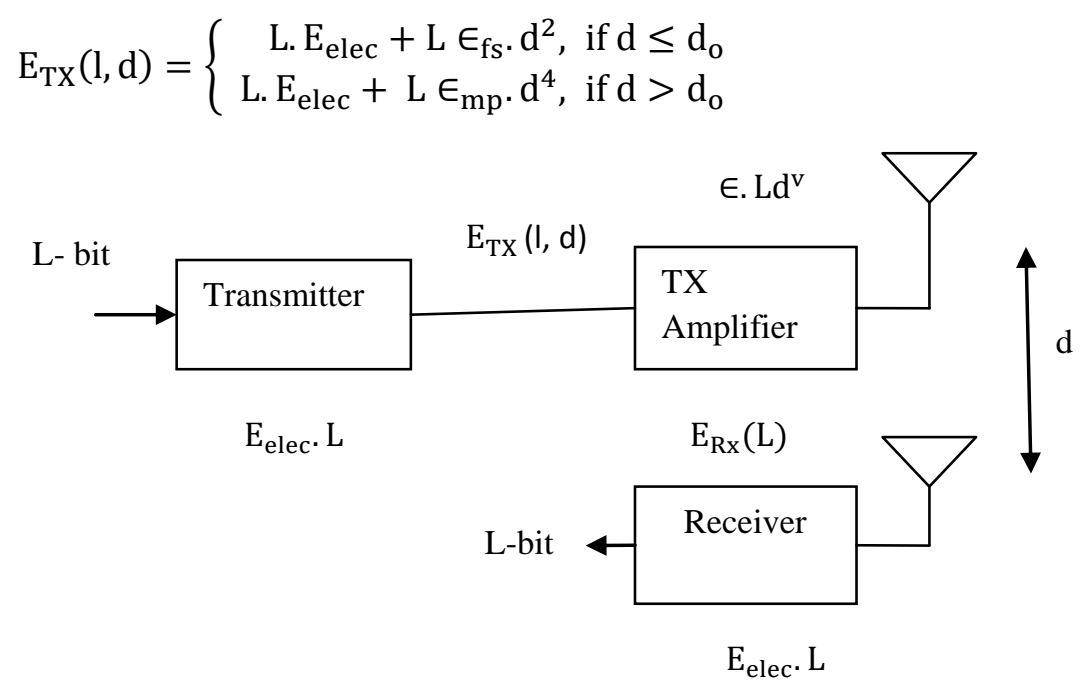

Figure 2. Radio dissipation model

Where $E_{\text {elec }}$ is measure of energy consumed by electronic circuit in sending data from transmitter side to receiver side. In this model both free space $\epsilon_{\mathrm{fs}}\left(\mathrm{d}^{2}\right.$ power loss) and multipath fading $\epsilon_{\mathrm{mp}}\left(\mathrm{d}^{4}\right.$ power loss) channels are used depending on distance between two communicating ends. Here $d_{o}$ is minimum (threshold) distance between communicating ends called as threshold distance calculated as

$$
\mathrm{d}_{\mathrm{o}}=\sqrt{\frac{\epsilon_{\mathrm{fs}}}{\epsilon_{\mathrm{mp}}}}
$$

\section{G-DEEC Protocol}

In this section, we present the detailed proposed protocol G-DEEC. Sensor nodes with two level of heterogeneity are considered i.e. $\mathrm{m} 1$ fraction of nodes called advance nodes are given ' $\alpha 1 \%$ additional energy as compared to normal nodes unlike DEEC where $m$ fraction are provided with $a \%$ additional energy. Sensor nodes are randomly distributed over the field with some intermediate nodes called gateway deployed on field at pre-determined distance covering the whole network field so that head of each cluster formed is near to that gateway so as to reduce the transmission distance from cluster head to base station with the help of these gateway nodes. Here we deploy 9 gateway nodes which are taken approximately on basis of number of cluster heads formed. 4 gateways are placed at four corners of sensor field and 4 gateways at center of four boundaries and one at center of field. Moreover the gateway nodes deployed are rechargeable proving to be back-up for exhausted network power sources. The function of gateway nodes to collect aggregated data from $\mathrm{CHs}$ and fuses this data and forward to base station. Sensor nodes near to gateway can transfer data directly to gateway nodes. At a time only one $\mathrm{CH}$ will transfer data to gateway node and incase of failure of one gateway node, other nearby gateway node can be used for data forwarding. The $\mathrm{CH}$ selection is done on basis of weighted election probabilities. The weighted election probability for normal nodes is as: 
$\mathrm{p}_{\mathrm{nrm}}=\frac{\mathrm{p}_{\mathrm{opt}}}{1+\mathrm{m} 1 \times \alpha 1} \times \frac{\mathrm{E}_{\mathrm{i}}}{\mathrm{E}_{\mathrm{a}}}$

The weighted election probability for advance nodes is as:

$p_{a d v}=\frac{p_{\text {opt }} \times(1+\mathrm{a} 1)}{1+\mathrm{m} 1 \times \mathrm{a} 1} \times \frac{\mathrm{E}_{\mathrm{i}}}{\mathrm{E}_{\mathrm{a}}}$

Where $E_{i}$ is residual energy of node and $E_{a}$ is average energy given as:

$$
\mathrm{E}_{\mathrm{a}}=\frac{1}{\mathrm{n}} \times \mathrm{E}_{\text {total }} \times\left(1-\frac{\mathrm{r}}{\mathrm{R}}\right)
$$

Where, $\mathrm{n}$ is total number of nodes, $\mathrm{E}_{\text {total }}$ is total energy, $\mathrm{r}$ is current round and $\mathrm{R}$ is total rounds of the network lifetime. Average energy is just used as reference energy for each node. It is the ideal required energy that each node should own in current round to keep the network alive to greatest extent. After election of $\mathrm{CH}$ having maximum energy; a single $\mathrm{CH}$ is selected per round for transmitting data. For this, a random number is generated and then comparing random number with threshold value. If random number is less than threshold value then it becomes $\mathrm{CH}$ for that particular round. The threshold is calculated differently for normal and advance nodes. Threshold function for normal nodes given as:

$$
\mathrm{T}\left(\mathrm{n}_{\mathrm{nrm}}\right)=\left\{\begin{array}{ll}
\frac{p_{n r m}}{1-\mathrm{p}_{\mathrm{nrm}}\left[\mathrm{r} \times \bmod \left(\frac{1}{\mathrm{pnrm}}\right)\right]}, & \text { if } \mathrm{n} \in \mathrm{g}^{\prime} \\
0 & \text { otherwise }
\end{array}\right\}
$$

Where $r$ is ongoing round number, g' is set of possible normal nodes that have not been cluster head in last $1 / \mathrm{P}_{\mathrm{nrm}}$ rounds.

Threshold function for advanced nodes given as:

$\mathrm{T}\left(\mathrm{n}_{\mathrm{adv}}\right)=\left\{\begin{array}{ll}\frac{\mathrm{p}_{\mathrm{adv}}}{1-\mathrm{p}_{\mathrm{adv}}\left[\mathrm{r} \times \bmod \left(\frac{1}{\mathrm{p}_{\mathrm{adv}}}\right)\right]} & , \text { if } \mathrm{n} \in \mathrm{g}^{\prime \prime} \\ 0 & \text { otherwise }\end{array}\right\}$

Where $r$ is ongoing round number, $g$ "' is set of possible advance nodes that have not been cluster head in last $1 / \mathrm{P}_{\mathrm{adv}}$ rounds. 


\section{PERFORMANCE EVALUATION}

\subsection{Network parameters}

Table I: Network Parameters

\begin{tabular}{|l|l|}
\hline $\mathrm{n}$ & 100 \\
\hline $\begin{array}{l}\mathrm{E}_{0} \text { (Initial energy } \\
\text { of normal nodes) }\end{array}$ & $0.5 \mathrm{~J}$ \\
\hline 1 (Message Size ) & $4000 \mathrm{bits}$ \\
\hline $\mathrm{E}_{\mathrm{elec}}$ & $50 \mathrm{~nJ} / \mathrm{bit}$ \\
\hline $\mathrm{E}_{\mathrm{DA}}$ & $5 \mathrm{~nJ} / \mathrm{bit} / \mathrm{signal}$ \\
\hline$\epsilon_{\mathrm{fs}}$ & $10 \mathrm{pJ} / \mathrm{bit} / \mathrm{m}^{2}$ \\
\hline$\epsilon_{\mathrm{mp}}$ & $0.0013 \mathrm{pJ} / \mathrm{bit}^{\mathrm{m}} \mathrm{m}^{4}$ \\
\hline $\mathrm{d}_{0}$ & $70 \mathrm{~m}$ \\
\hline $\mathrm{r}$ (No. of Rounds) & 2000 \\
\hline $\mathrm{m} 1$ & $30 \%$ \\
\hline$\alpha 1$ & $300 \%$ \\
\hline $\mathrm{m}$ & $20 \%$ \\
\hline$a$ & $350 \%$ \\
\hline
\end{tabular}

Simulation of our proposed protocol is done in MATLAB. We consider a $100 \mathrm{~m} * 100 \mathrm{~m}$ field with $\mathrm{n}$ sensor nodes deployed randomly and 9 rechargeable gateway nodes. We ignore the effects of signal inference and collision. Radio characteristics and other parameters are given in Table I above.

\subsection{Performance parameters}

5.2.1 Network lifetime: It is defines as time interval till the network is operational which is calculated based on number of half dead nodes and alive nodes.

5.2.1.1 Half dead Nodes- It is defined when sensor nodes had spent half of their total energy i.e. it is partially dead and partially alive (i.e. energy $\leq \frac{E_{0}}{2} \&>0$ ). These partially alive nodes can still be part of network operation and thereby increases the lifetime of network. Greater the number of half dead nodes, better the lifetime of network.

5.2.1.2 Alive Nodes- It is defined as sensor nodes that still have not used all of their energy even after processing (i.e. energy $>0$ ). Greater the number of alive nodes more is the lifetime of the network. 
International Journal on Cybernetics \& Informatics (IJCI) Vol. 4, No. 5, October 2015

5.2.1.3 Dead Nodes- Sensor nodes are considered dead after they consume all of its initial energy (i.e. energy < 0) provided to them i.e. they are of no use in network processing. Lesser the number of dead nodes more is the lifetime of the network.

\subsubsection{Stability Period (First Node Dead):}

It is defined as the time interval between start of network operation till the death of first sensor node. Later the first dead node comes, greater the stability period.

5.2.3 Residual Energy: It is defined as measure of total remaining energy in the network. It is used to analyze the energy consumption of sensor nodes in each round. Lesser the energy consumption better is the protocol.

\subsection{Simulation Results and Analysis}

5.3.1 Network lifetime: Simulation results for number of half dead nodes, alive nodes and dead nodes are considered for analyzing the lifetime improvement. In DEEC, $20 \%$ of nodes are given $350 \%$ additional energy whereas in proposed G-DEEC, $30 \%$ of nodes are given $300 \%$ additional energy. In figure 3, we show the results for number of half dead nodes. Nodes are considered dead after consumption of full 0.5 joule initial energy but they are half dead when they have not consumed their all of initial energy. At round 2000, number of half dead nodes are 0 whereas they are coming out to be 30 in case of proposed G-DEEC. More the number of half dead nodes more is the network lifetime.

In figure 4, we show the simulation results of number of alive nodes. At round 2000, number of alive nodes are 20 in DEEC whereas this number has increased to 61 in case of proposed GDEEC. Greater the number of alive nodes greater is the lifetime of network.

Figure 3 and 4 figure shows the increment in number of half dead nodes and number of alive nodes in proposed G-DEEC, making the network to be operational for longer time as compare to single hop DEEC. Half Dead Nodes shows 30 \% improvement w.r.t. initial 100 nodes whereas alive nodes shows $41 \%$ improvement w.r.t initial 100 deployed nodes. Both parameters combined shows $35.5 \%$ improvement in network lifetime.

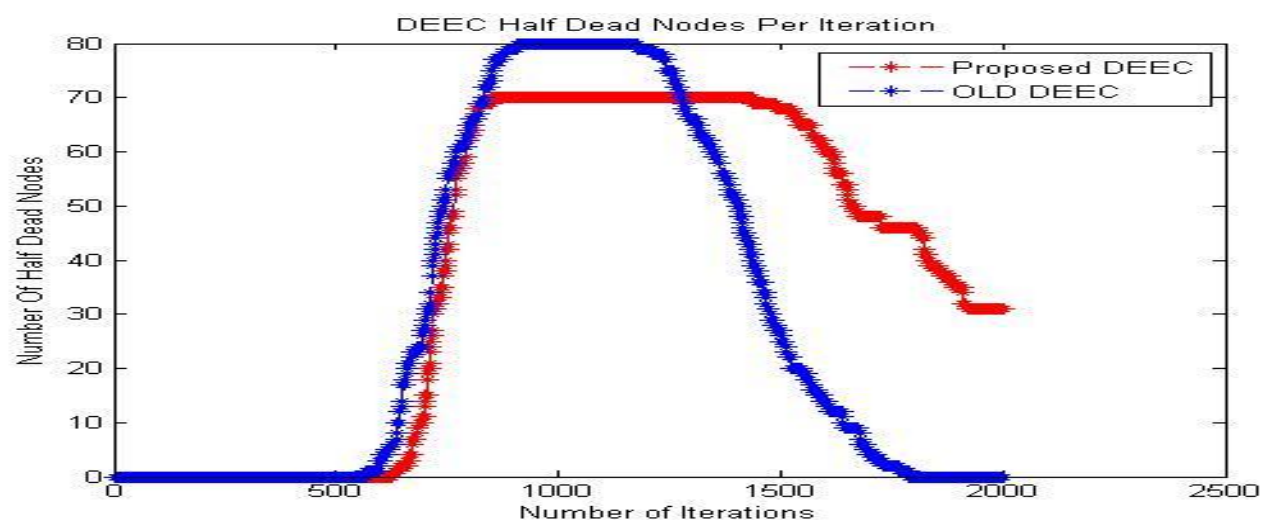


International Journal on Cybernetics \& Informatics (IJCI) Vol. 4, No. 5, October 2015

Figure 3. Number of Half Dead Nodes

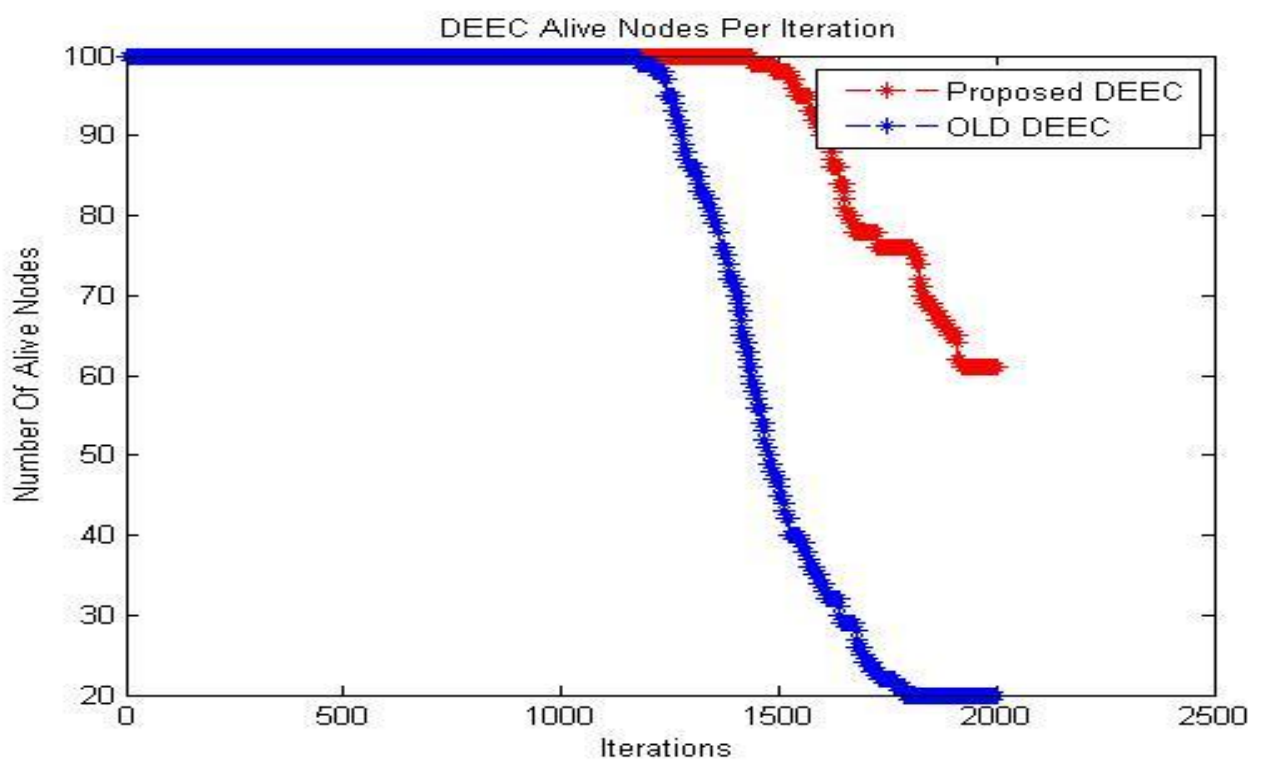

Figure 4. Number of alive nodes

Figure 5 shows the plot of number of dead nodes at round 2000. The number of dead nodes at round 2000 is 80 in old DEEC whereas they are coming to be 39 in proposed G-DEEC. There is decrement in number of dead nodes making the network processing time longer thereby increasing the network lifetime of sensor network. Both the plots of alive nodes and dead nodes are vice-versa to each other. 
International Journal on Cybernetics \& Informatics (IJCI) Vol. 4, No. 5, October 2015

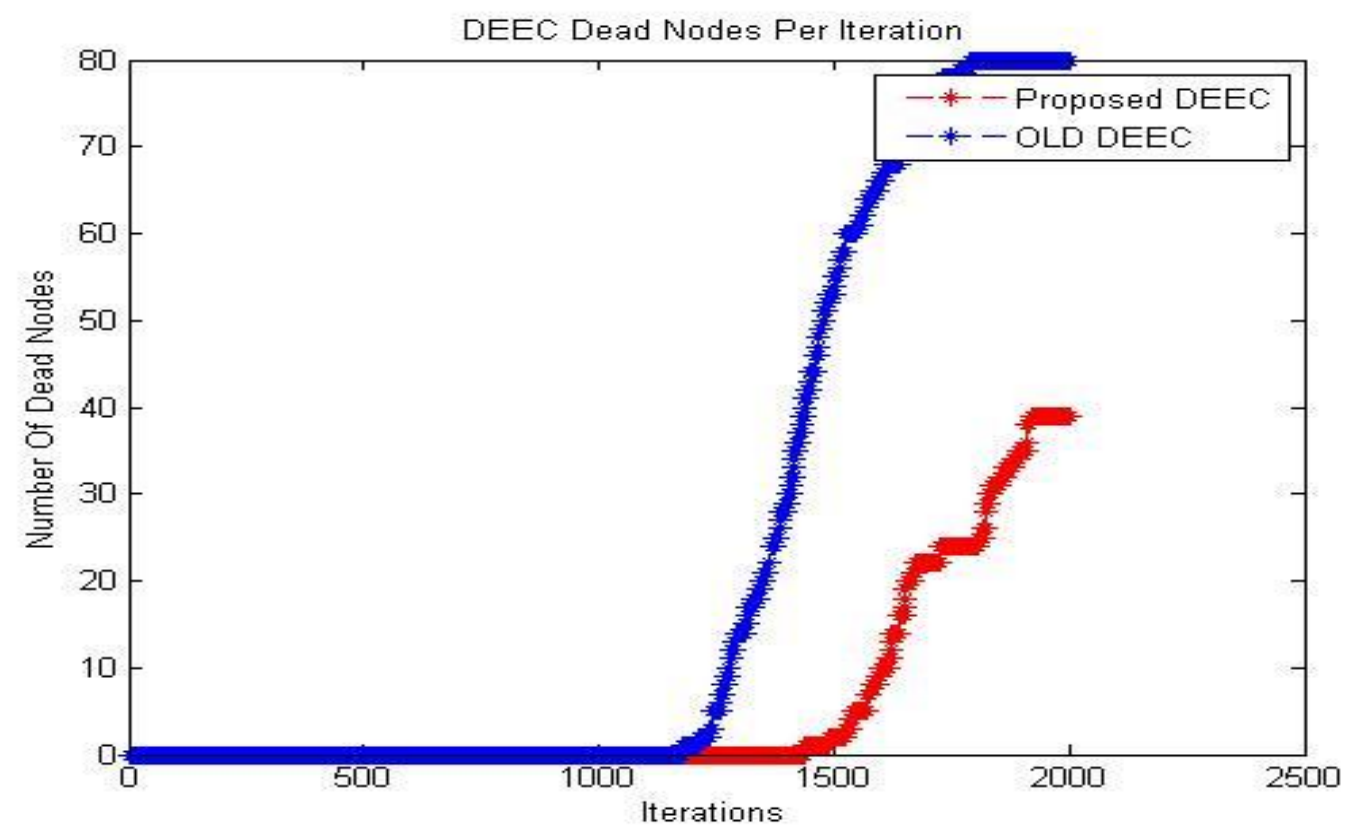

Figure 5. Number of dead nodes

TABLE II Improvement table of network lifetime in G-DEEC

\begin{tabular}{|l|c|c|c|}
\hline Operation at 2000 iteration & $\begin{array}{c}\text { DEEC } \\
\text { (Improvement } \\
\text { w.r.t. initial } \\
\text { values) }\end{array}$ & $\begin{array}{c}\text { G-DEEC } \\
\text { (Improvement } \\
\text { w.r.t. initial } \\
\text { values) }\end{array}$ & $\begin{array}{c}\text { Overall } \\
\text { Improvement }\end{array}$ \\
\hline Number of Half Dead Nodes & $0(0 \%)$ & $30(30 \%)$ & $30 \%$ \\
\hline Number of Alive Nodes & $20(20 \%)$ & $61(61 \%)$ & $41 \%$ \\
\hline Number of Dead Nodes & $80(20 \%)$ & $39(61 \%)$ & $41 \%$ \\
\hline
\end{tabular}

5.3.2 Stability Period (First Node Dead): Simulation results of first node dead are shown in figure 6. The first node coming to be dead at about 1178 in DEEC whereas it is becoming dead at 1438 in proposed G-DEEC. The first node in proposed G-DEEC dies after 260 processing rounds as compared to DEEC. This leads to increase in stability of network by $13 \%$. 
International Journal on Cybernetics \& Informatics (IJCI) Vol. 4, No. 5, October 2015

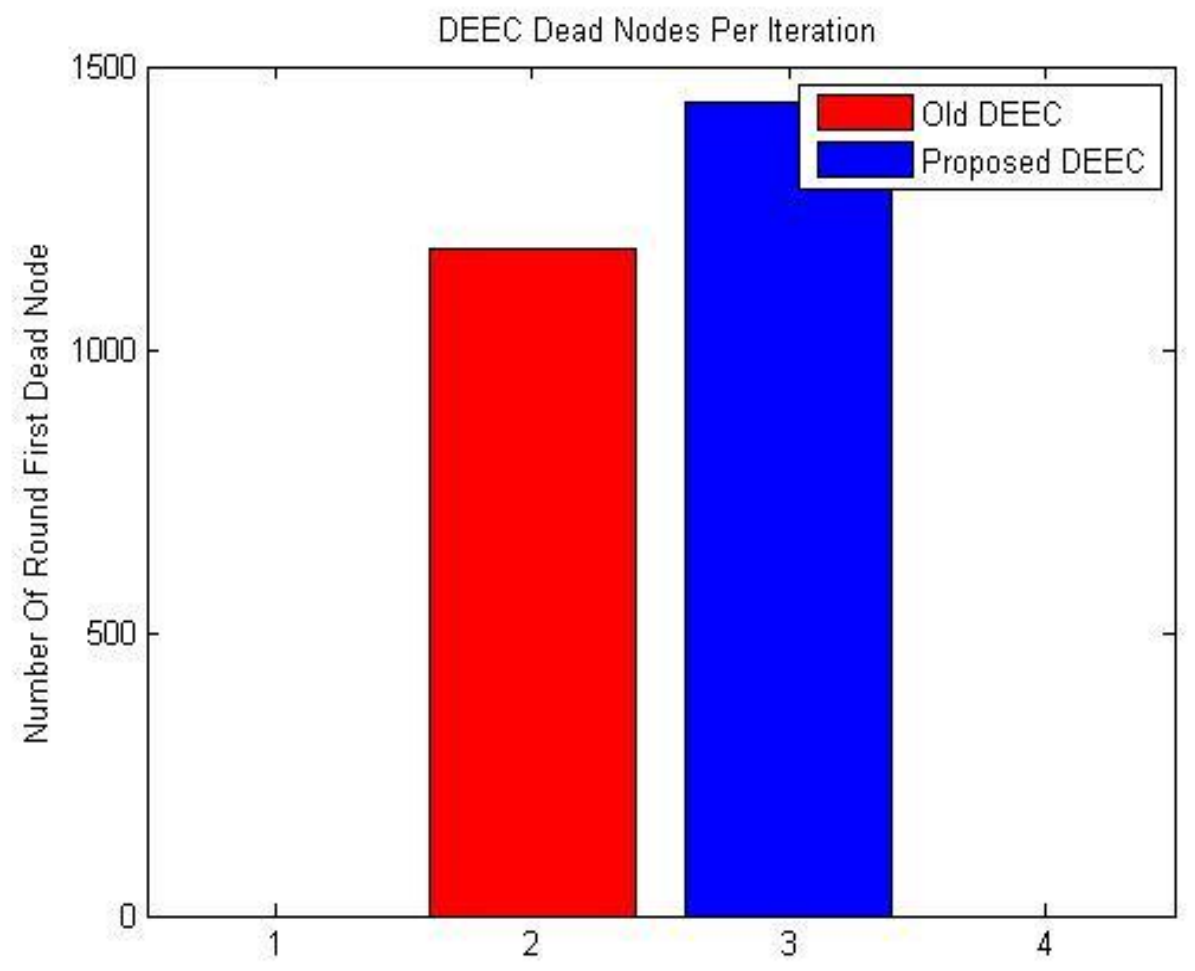

Figure 6. Interval plot of first node dead

5.3.3 Residual Energy: The plot of energy consumption per round is considered in figure 7. At round 1500, energy remained per round is about zero joules in DEEC whereas it is about 0.1 joules in proposed G-DEEC. Thereby energy consumption is reduced in proposed protocol as shown in figure7. So network will remain operational for longer time with same battery power in proposed G-DEEC protocol as compared to old DEEC protocol. So the rechargeable gateways proved to be beneficent in reducing the energy consumption of sensor nodes. 


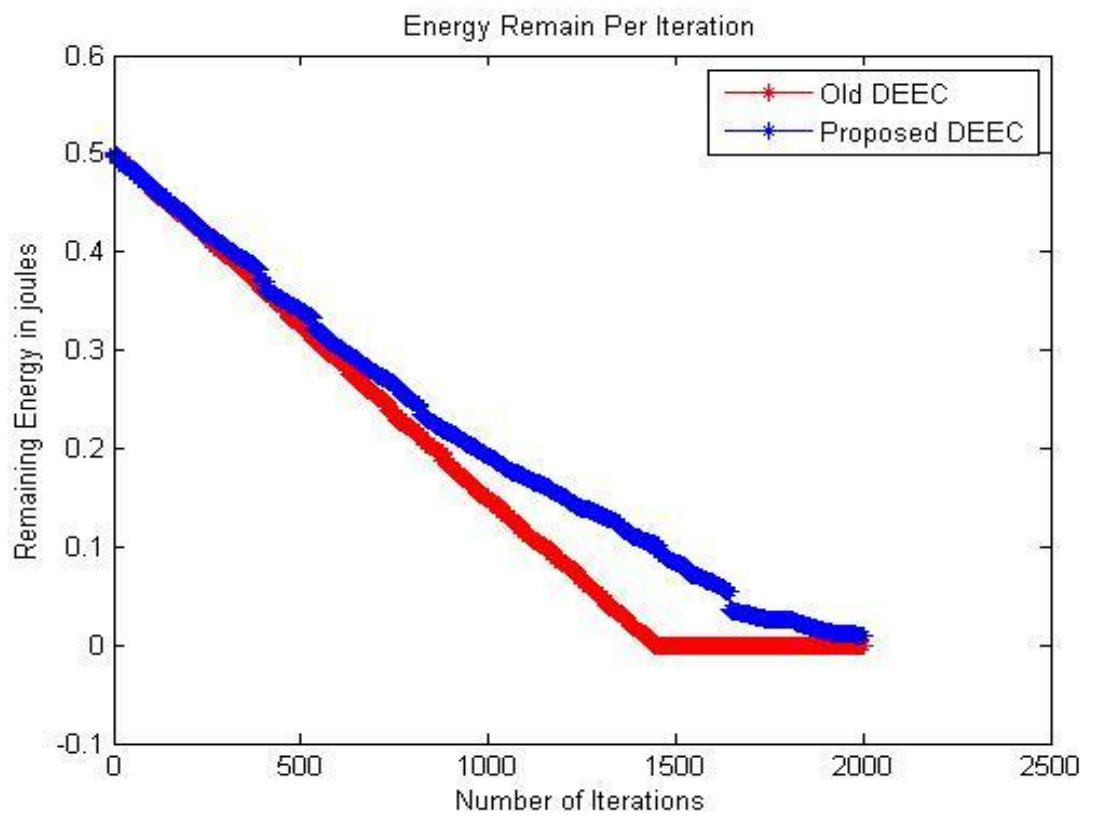

Figure7. Analysis of remaining energy

TABLE III Improvement table of stability and residual energy in G-DEEC

\begin{tabular}{|l|c|c|c|}
\hline \multicolumn{1}{|c|}{ Operation at 2000 iteration } & $\begin{array}{c}\text { DEEC } \\
\text { (Improvement } \\
\text { w.r.t. initial } \\
\text { values) }\end{array}$ & $\begin{array}{c}\text { G-DEEC } \\
\text { (Improvement } \\
\text { w.r.t. initial } \\
\text { values) }\end{array}$ & Overall \\
Improvement \\
\hline First Dead Node (Stability Period) & $1178(58.9 \%)$ & $1438(71.9 \%)$ & $13 \%$ \\
\hline Residual energy (joules) at round & $0(0 \%)$ & $0.1(20 \%)$ & $20 \%$ \\
\hline
\end{tabular}

\section{CONCLUSIONS AND FUTURE SCOPE}

In this research, an energy aware energy efficient G-DEEC has been proposed. Number of half dead nodes, number of alive nodes and number of dead nodes in proposed G-DEEC protocol shows $30 \%, 41 \%, 41 \%$ overall improvement respectively with respect to DEEC, thus showing $37.33 \%$ overall improvement on an average. The first sensor node dying much later in proposed G-DEEC as compared to DEEC showing $13 \%$ overall improvement in stability of network. There is $20 \%$ overall improvement in residual energy. This work can further be extended to multi-level heterogeneity i.e. inclusion of super nodes and other intermediate nodes of different energy and can also be implemented on ETX metric scheme. 
International Journal on Cybernetics \& Informatics (IJCI) Vol. 4, No. 5, October 2015

\section{REFERENCES}

[1] I.F. Akyildiz, W.Su, Y. Sankarasubramaniam and E. Cayirci, "A Survey on sensor networks", IEEE Communication Magazine, vol. 40, no. 8, pp. 102-114, 2002.

[2] W.R. Heinzelman, A. Chandrakasan and H. Balakrishnan, "Energy-efficient communication protocol for wireless microsensor networks", In Proc. International Conference on System Sciences, pp.4-7, Mauii, Hawaii, 2000.

[3] S. Lindsey and C.S. Raghavendra, "PEGASIS: Power-Efficient Gathering in Sensor Information Systems", IEEE Proceedings on Aerospace, vol. 3, no.2, pp.1-6, 2002.

[4] O. Younis and S. Fahmy, "HEED: A hybrid, energy efficient, distributed clustering approach for ad hoc sensor networks", IEEE Transactions on Mobile Computing, vol. 3, no. 4, pp. 660-669, 2004.

[5] G. Smaragdakis, I. Matta and A. Bestavros, "SEP: A Stable Election Protocol for Clustered Heterogeneous Wireless Sensor Networks", In Proc. of IEEE International Workshop on SANPA, pp. 1-11, Boston, 2004.

[6] L. Qing, Q. Zhu and M. Wang, "Design of a distributed energy-efficient clustering algorithm for heterogeneous wireless sensor networks", Journal of Computer Communications, vol. 29, no.4, pp. 2230-2237, 2006.

[7] B. Elbhiri, S. Rachid, S. El Fkihi and D. Aboutajdine, "Developed distributed energy-efficient clustering (DDEEC) for heterogeneous wireless sensor networks", In proc. IEEE 5th International Symposium on I/V Communications and Mobile Network, pp. 1-4.,Rabat, 2010.

[8] P. Saini and A. K. Sharma, "E-DEEC- Enhanced distributed energy efficient clustering scheme for heterogeneous WSN", In proc. IEEE 1st International Conference on Parallel, Distributed and Grid Computing, pp. 205-210, Solan, 2010.

[9] M. Kaur, A. Jain and A.K. Goel, "Energy efficient two level distributed clustering scheme to prolong stability period of wireless sensor network", In proc. IEEE International Conference on Advances in Computing, Communication and Informatics, pp. 68-73, New Delhi, India, 2014.

[10] P. Jain, H. Kaur, "Gateway based stable election multi hop routing protocol for wireless sensor networks. International Journal of Mobile Network Communications \& Telematics, vol. 4, no.5, 2014. in Computing, Communication and Informatics, pp. 19-33, New Delhi, India,2014

[11] M.Y. Wang, J. Ding, W.P. Chen and W.Q. Guan,"SEARCH: A stochastic election approach for heterogeneous wireless sensor networks", IEEE Communication letters, vol.9, no.3, pp. 443-446, 2015.

[12] D. Kumar, T.C. Aseri and R.B Patel, "EEHC: Energy efficient heterogeneous clustered scheme for Wireless Sensor Networks", Journal of Computer Communication, vol.32, no. 3, pp. 662-667, 2009

[13] P. Saini and A. K. Sharma, "Energy efficient scheme for clustering protocol prolonging the lifetime of heterogeneous wireless sensor networks", International Journal of Computer Applications, vol.6, no.2, pp. 30-36, 2010

[14] S. Singh, A.K. Chauhan, S. Raghav, V. Tyagi and S. Johri, "Heterogeneous protocols for increasing lifetime of wireless sensor networks", Journal of Global Research in Computer Science, vol.2, no. 4, pp.172-176, 2011.

[15] S.B. Alla, A. Ezzati, A. Mouhsen, A.B. Hssane and M.L. Hasnaoui, "Balance and centralized energy efficient clustering for heterogeneous wireless sensor networks", In proc. IEEE 3rd International conference on next generation networks and services, pp .39-44, Hammamet, 2011.

[16] R. Meelu and R. Anand, "Performance evaluation of cluster-based routing protocols used in heterogeneous wireless sensor networks", International Journal of Information Technology and Knowledge Management, vol. 4, no. 4, pp. 227-231, 2011.

[17] M.M. Islam, M.A. Matin, and T.K Mondol, "Extended stable election protocol for three-level hierarchical clustered heterogeneous WSN", In Proc. of IET Conference on Wireless Sensor Systems, pp. 1-4, London, England, 2012. 
International Journal on Cybernetics \& Informatics (IJCI) Vol. 4, No. 5, October 2015

[18] Q. Nadeem, M.B. Rasheed, N. Javaid, Z.A Khan, Y. Maqsood and A. Din, "M-GEAR: GatewayBased Energy-Aware Multi-Hop Routing Protocol for WSNs", In proc. IEEE International Conference on Broadband, Wireless Computing, Communication and Applications, pp. 164-169, Compiegne, France, 2013.

[19] Z. Kaili, Z. Lihua, Wang Xu, "An Improved DEEC Algorithm with Isolated nodes", In proc. International conference on Information and Communication Technologies IETICT, pp. 228-232, Beijing 2013.

[20] N. Javaid, M. Waseem, Z. A. Khan, U. Qasim, K. Latif and A. Javaid, ”ACH: Away cluster heads scheme for energy efficient clustering protocols in WSNs", In proc. IEEE Saudi International Conference on Electronics, Communications and Photonics, pp.1-4, Fira, 2013.

[21] R. Tandon and S. Nandi, "CRP: Cluster head Re-election protocol for heterogeneous wireless sensor networks", In proc. IEEE 5th International conference on communication systems and networks, pp. 1-10, Bangalore, 2013.

[22] A.A. Malluh, M.K. Elleithy, Z. Qawaqney, J.R. Mstafa and A. Alanazi, "EM-SEP: An Efficient Modified Stable Election Protocol", In proc. IEEE Conf. of American Society for Engineering Education, pp. 1-7, Bridgeport, Connecticut, USA, 2014.

\section{AUTHORS}

SHEENAM received her Bachelor's of Technology in Electronics and Communication Engineering from Swami Vivekanand Institute of Engineering \&Technology, Banur affiliated to Punjab Technical University, Jalandhar, Punjab, India in 2012, and completed her degree in Masters of Technology in Electronics and Communication Engineering from Punjabi University, Patiala, Punjab, India in 2015. She yet has published two papers on wireless sensor networks. Her research interests include routing and energy management in

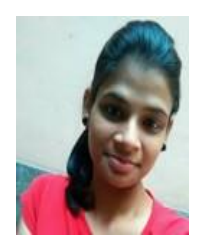
wireless sensor networks etc. 